\title{
Taste and Bulimia
}

\author{
ADAM DREWNOWSKI ${ }^{1}$ \\ Human Nutrition Program, School of Public Health and Department of Psychiatry \\ Medical School, The University of Michigan, Ann Arbor, MI 48109 \\ FRANCE BELLISLE \\ Laboratoire de Neurobiologie de la Nutrition, Ecole Pratique des Hautes Etudes \\ College de France, Paris 75231 \\ PIERRE AIMEZ \\ Departement de Medicine et Nutrition, Hotel-Dieu, Parvis Notre-Dame, Paris 75004 \\ AND \\ BRIGITTE REMY \\ Department de Psychiatrie, Mutuelle Generale de l'Education Nationale, Paris
}

Received 9 June 1987

\begin{abstract}
DREWNOWSKI, A., F. BELLISLE, P. AIMEZ AND B. REMY. Taste and bulimia. PHYSIOL BEHAV 41(6) 621-626, 1987.-Binge-eating episodes in bulimia often involve sweet or fat-containing foods. Sensory perceptions and preferences for sweetness and fat content were examined in 16 normal-weight women with a diagnosis of DSM IIIR bulimia and in 16 normal-weight volunteer controls. Taste stimuli were 15 semi-liquid mixtures of dessert-type soft white cheese ("fromage blane") containing 0,3 or 7 grams of fat per $100 \mathrm{~g}$, and sweetened with $1,5,10,20$, or $40 \%$ sucrose (wt./wt.). The subjects used 9-point category scales to rate the perceived sweetness and fat content of the stimuli, and assigned a pleasantness (hedonic) rating to each sample. Taste preferences were modelled using the Response Surface Method (RSM). Mean estimates of sweetness intensity and fat content were generally similar for bulimic patients and controls. In contrast, profiles of taste preference differed significantly between groups. Opitmal stimulus sweetness was $15 \%$ sucrose wt./wt. for bulimic patients and only $9 \%$ for controls, while optimal fat levels were lower for bulimic patients relative to controls. The present data are consistent with previous reports that patients with eating disorders crave sweetness but show reduced sensory preferences for fat-containing foods.
\end{abstract}

Fat and sugar Sensory evaluation Hedonic preference Response surface method Eating disorders

THE syndrome of bulimia is characterized by frequent and uncontrolled binge-eating episodes that are followed by fasting, purging or self-induced vomiting [2]. It is estimated that between 1 and $3 \%$ of young women suffer from this eating disorder $[12,15]$, often alternating food binges with periods of rigid dieting.

Binge-eating has been viewed as a form of addictive behavior. Bulimic patients describe binges as bouts of overeating they are unable to control [1]. The sensation of satiety is absent, and eating is reported to be accompanied by racing pulse, sweating, breathlessness and other symptoms of sympathetic activation [18]. Precipitating events may include tension, being alone, craving specific foods (often sweets), thinking of food or actually eating something [1]. Bulimics often seem to know that a binge is imminent, and there are reports of hoarding, buying and preparing foods in anticipation [1]. Revulsion and disgust tend to follow eating binges. Almost invariably, patients induce vomiting immediately after a binge in an effort to regurgitate swallowed food and get rid of unwanted calories [19].

The choice of "binge foods" is also of considerable interest. Bulimic patients often say that they eat too quickly during a binge to taste anything [1]. However, all patients feel that the taste and texture of food are important, at least at the beginning of the binge. Among the principal binge foods are reported to be ice cream, cookies, cake, chocolate and other sweet desserts $[1,19,22]$. Some bulimics report binges on non-sweet but fat-containing foods, especially cheese, yogurt, and other dairy products. Monitoring binge-eating behavior in a hospital setting showed that the patients mostly ate doughnuts, pies, sandwiches, and chocolate candy [19]. In contrast, eating bouts not classified as binges included salads and diet soft drinks.

The patients' food choices during a binge provide a striking contrast to their usual diets. Young women with eating disorders are reported to avoid all fattening foods, notably

'Requests for reprints should be addressed to Adam Drewnowski, School of Public Health, University of Michigan, Ann Arbor, MI 48109. 
TABLE 1

DSM IIIR CRITERIA FOR BULIMIA

A. Recurrent episodes of binge eating

B. Fear of not being able to stop eating during a binge

C. Regular use of self-induced vomiting, laxatives, fasting or rigorous dieting to counteract the effects of binge eating

D. Minimum average of two binge eating episodes per week for at least three months

TABLE 2

SUMMARY OF SUBJECT CHARACTERISTICS

\begin{tabular}{lrr}
\hline & $\begin{array}{c}\text { Bulimics } \\
(\mathrm{n}=16)\end{array}$ & \multicolumn{1}{c}{$\begin{array}{c}\text { Controls } \\
(\mathrm{n}=16)\end{array}$} \\
\hline Weight $(\mathrm{kg})$ & $56.7(1.6)$ & $55.8(0.9)$ \\
Height $(\mathrm{cm})$ & $165.1(1.4)$ & $164.4(1.0)$ \\
BMI $\left(\mathrm{kg} / \mathrm{m}^{2}\right)$ & $20.9(0.6)$ & $20.7(0.3)$ \\
Age (years) & $26.7(2.0)$ & $28.9(1.8)$ \\
\hline
\end{tabular}

The data are means (and SEMs).

sugar and other carbohydrates $[13,23]$ as well as foods rich in fat [3]. Yet the same "forbidden" sweet foods are consumed to excess during a binge, in vast amounts, rapidly, and in private. Clinicians estimate that a typical binge involves between $3-5,000 \mathrm{kcal}$ and lasts about an hour [16]. One report [19] cited the average amount of calories eaten per binge as $4,394 \mathrm{kcal}$ (range $1,436-8,585 \mathrm{kcal}$ ) and the mean binge duration as 59 minutes.

Clinicians and patients agree that eating binges are extremely difficult to control. Past studies have interpreted binge-eating in terms of breakdown of dietary restraint [21] and counterregulation. However, the reported awareness of an impending binge, loss of control, and frequent (though not invariable) choice of sweet foods suggest that bulimic episodes may have a physiological as we"l as a psychological basis. Specifically, it may be that binge-eating episodes in anorexia nervosa and bulimia are linked to an uncontrollable craving for sweet taste.

In previous studies by Drewnowski and co-workers [611], human preferences for sweetened high-fat foods were examined as a function of body-weight status. Obese women preferred stimuli rich in fat to those that were intensely sweet [8]. Conversely, emaciated anorectic and bulimic young women preferred very sweet stimuli to those containing butterfat [11]. In the present study, we compared taste responses of normal-weight bulimics with those of normalweight control women. Bulimia was characterized by frequent binge eating followed by self-induced vomiting. Sensory responsiveness to sugar and fat was examined using a model system consisting of semi-liquid white cheese sweetened with different amounts of sucrose.

\section{METHOD}

\section{Subjects}

Sixteen normal-weight women with a clinical diagnosis of DSM IIIR bulimia took part in the study. The DSM IIIR diagnostic criteria are summarized in Table 1. All patients reported multiple binge-purge episodes per week for at least
TABLE 3

STIMULUS COMPOSITION PER $100 \mathrm{~g}$ OF DAIRY PRODUCT

\begin{tabular}{lccccc}
\hline $\begin{array}{l}\text { Stimulus } \\
\text { (Fat as \% solids) }\end{array}$ & Kcal & $\begin{array}{c}\text { Water } \\
(\mathrm{g})\end{array}$ & $\begin{array}{c}\text { Fat } \\
(\mathrm{g})\end{array}$ & $\begin{array}{c}\text { Protein } \\
(\mathrm{g})\end{array}$ & $\begin{array}{c}\text { CHO } \\
(\mathrm{g})\end{array}$ \\
\hline & & & & & \\
$0 \%$ Fat & 48 & 87 & 0 & 8 & 4 \\
$20 \%$ Fat & 84 & 85 & 3 & 8 & 4 \\
$40 \%$ Fat & 110 & 80 & 7 & 8 & 4 \\
\hline
\end{tabular}

TABLE 4

SUMMARY OF EXPERIMENTAL DESIGN

Fat Content

$(\mathrm{g} / 100 \mathrm{~g}) \quad$ Sugar Content $(\% \mathrm{wt} / \mathrm{wt}$.

\begin{tabular}{llllll}
\hline 0 & 1 & 5 & 10 & 20 & 40 \\
3 & 1 & 5 & 10 & 20 & 40 \\
7 & 1 & 5 & 10 & 20 & 40 \\
\hline
\end{tabular}

three months prior to admission, and half reported several binge-purge episodes per day. Over half of the patients were amenorrheic or oligomenorrheic at the time of the study. All were admitted as outpatients to the psychiatry clinic at the Hotel-Dieu Hospital in Paris for a behaviorally-oriented program that included individual psychotherapy and nutritional counseling. Sensory testing was part of the initial evaluation protocol and was conducted shortly following hospital admission. The study was conducted on a volunteer basis and informed consent was obtained from each participant.

An additional control group of 16 normal-weight female subjects who were free of eating disorders was recruited from among students at the University of Paris and the staff of the College de France. The two groups were comparable in age, height and weight, as well as educational and socioeconomic status.

Subject characteristics are summarized in Table 2. Bulimic women weighed a mean of $56.7 \mathrm{~kg}$, while control women weighed a mean of $55.8 \mathrm{~kg}$. Their mean body mass indices $\left(\mathrm{BMI}=\mathrm{wt} . / \mathrm{ht}^{2}\right)$ were comparable. Body mass index of 20.9 corresponds exactly to the median value of BMI for 21 -year-old women as indicated by growth charts prepared by the French National Institute for Health and Medical Research (INSERM).

\section{Procedures}

The stimuli were three different types of commercially available dessert-type soft white cheese ("fromage blanc") containing different amounts of butterfat. The cheese is marketed in France by Gervais Co. and is advertised as containing 0,20 , or $40 \%$ fat. These percentages refer to percent fat as solids weight by weight. According to the manufacturer's specifications, the three types of white cheese contain 0,3 and 7 grams of fat respectively per $100 \mathrm{~g}$ of product. They will be referred to as $0 \mathrm{~g}, 3 \mathrm{~g}$, and $7 \mathrm{~g}$ fat throughout. The three varieties of cheese also contain approximately $8 \mathrm{~g}$ protein and $4 \mathrm{~g}$ endogenous carbohydrate per $100 \mathrm{~g}$, and are very similar in terms of consistency and texture. Nutrient composition of white cheese is summarized in Table 3. Added sucrose levels were set at 1, 5, 10, 20, and $40 \%$ wt./wt. to produce 15 sensory stimuli in a $3 \times 5$ factorial design as summarized in Table 4. 


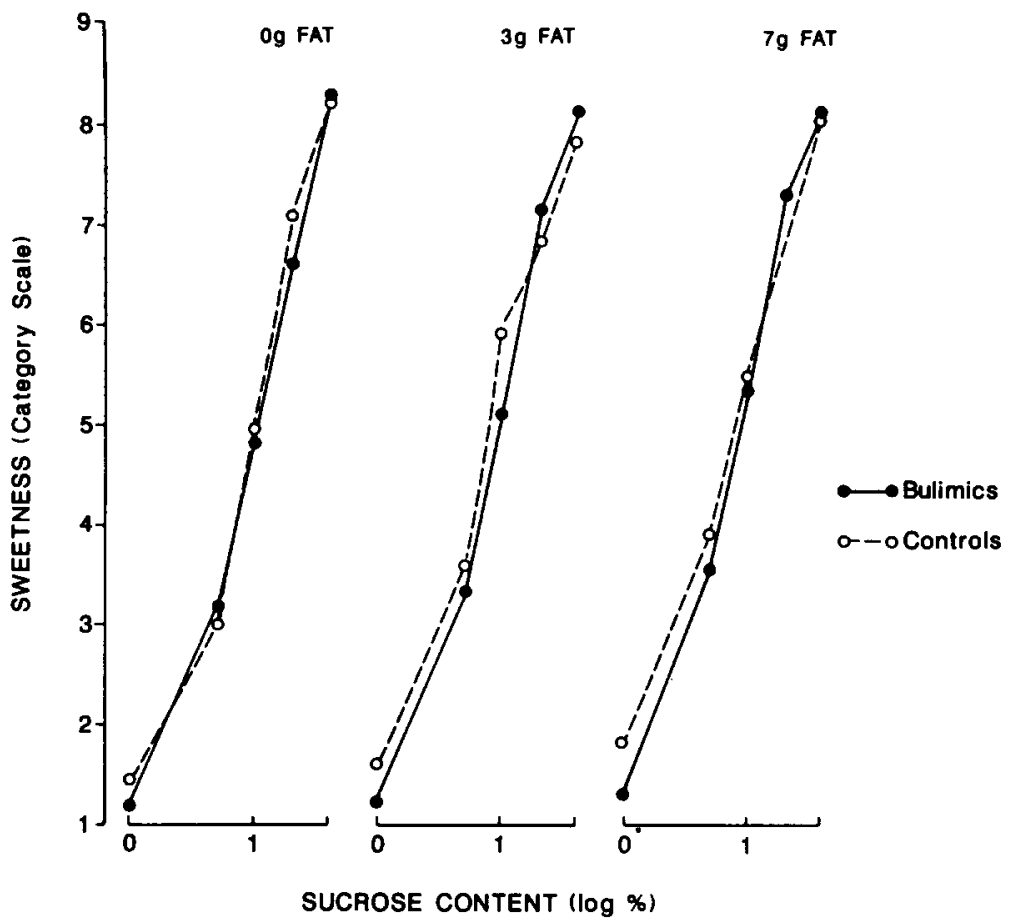

FIG. 1. Mean estimates of stimulus sweetness as a function of sucrose content for each type of dairy product. Sucrose levels are expressed as log percent wt./wt. Bulimics $(\bullet)$, controls $(O)$.

The taste samples were presented to subjects at $5^{\circ} \mathrm{C}$ in 30 ml plastic cups and in a random order. Subjects used a spoon to taste each stimulus, keeping it in the mouth for as long as needed to make judgments of sensory intensity and hedonic preference. The subjects would then spit out the sample and rinse mouth with tap water. Each sample was rated along 9-point category scales. Hedonic response was measured along a scale that ranged from "extremely unpleasant" to "extremely pleasant." Sweetness intensity was measured along a 9-point scale ranging from "not at all sweet" to "extremely sweet," while fat conent was measured along a scale ranging from "not at all fat" to "extremely fat." All subjects were tested in the morning, approximately 3 hours following a standard breakfast.

\section{Data Analyses}

Intensity estimates of sweetness and fat content were analyzed using BMDP ANOVA for repeated measures. Hedonic response profiles were analyzed using two principal dependent variables: the magnitude of the pleasantness response (analyzed using BMDP ANOVA), and the ingredient composition of the maximally pleasant (i.e., best tasting) mixture. In addition, a measure of inter-subject variability was obtained by plotting the frequency distribution of each subject's maximal pleasantness scores as a function of stimulus sucrose and fat levels. Frequency distribution of peak hedonic responses shown by bulimic patients and normal controls was then compared using Chi-square test.

Ingredient composition of mixtures rated by individual subjects as best-tasting was derived using a multivariate analytical procedure known as the Response Surface Method [6-11]. Fifteen hedonic scores obtained for each individual served as input for the RSM model. The hedonic response was assumed to be a non-linear function of the two sensory components-sugar and fat. For the present system, each of the 15 hedonic responses $(\mathrm{H})$ was assumed to be a function of sucrose $(\mathrm{S})$ and fat $(\mathrm{F})$ levels such that:

$$
\begin{aligned}
H=a_{0} & +a_{1}(\log S)+a_{2}(\log F) \\
& +a_{3}(\log S)^{2}+a_{4}(\log F)^{2} \\
& +a_{5}(\log S)(\log F)
\end{aligned}
$$

After coefficient values $a_{0}$ through $a_{5}$ were determined by solving 15 simultaneous equations, this algorithm was used to predict hedonic responses to a variety of sucrose (S) and fat $(F)$ levels, including those not actually tested. The model interpolated predicted data points among the 15 empirically obtained ones to yield a more accurate representation of the hedonic response surface than could be obtained from experimental data alone. Ingredient composition of the best-liked stimulus was then obtained by determining the sugar $(S)$ and fat $(F)$ levels that contributed to the maximum pleasantness score for a given individual. This mathematical modelling technique is especially useful when subjects assign comparable ratings to several stimuli and the location of the optimal response cannot be unambiguously determined from raw data. Goodness of fit of the model was determined by the least squares method.

\section{RESULTS}

\section{Taste Responsiveness}

Sensory perceptions of sweetness and fat content were comparable for bulimic patients and volunteer controls. However, sensory preferences were significantly different for the two groups, with bulimic patients showing optimum preferences for stimuli that were sweeter but low in fat content. 


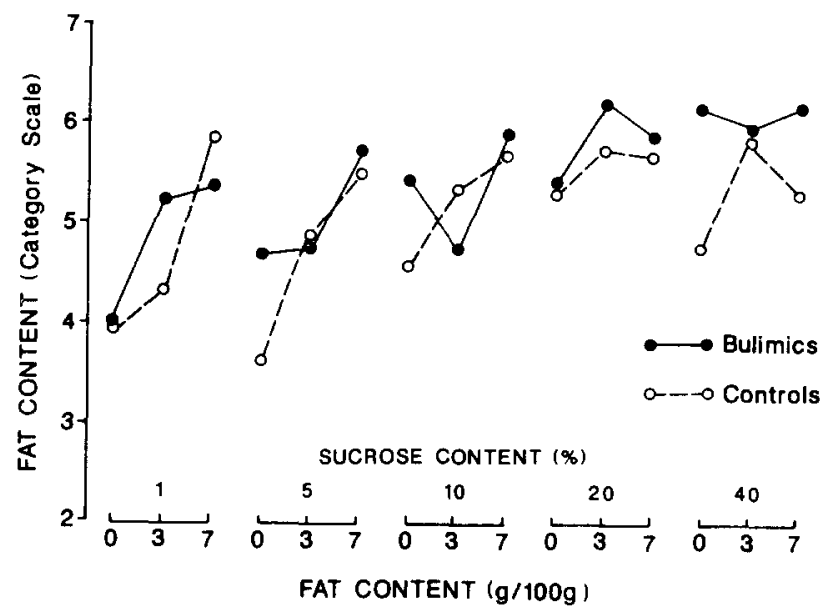

FIG. 2. Mean estimates of stimulus fat content at each level of sucrose. Fat content is expressed as grams per $100 \mathrm{~g}$ (wt./wt.).

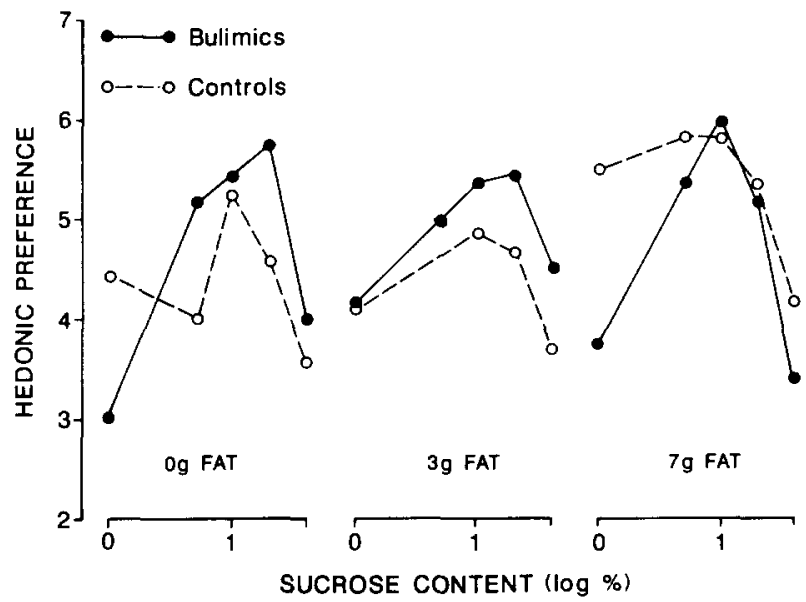

FIG. 3. Mean hedonic ratings as a function of sucrose content for each type of dairy product.

Intensity estimates for sweet taste are summarized in Fig. 1. Perceived sweetness increased as a monotonic function of sucrose levels. The main effect of sucrose was highly significant, $\mathrm{F}(2,60)=332.68, p<0.01$. There was no main effect of diagnostic group and no significant interactions. However, more detailed analyses revealed that bulimic women consistently rated the lowest sucrose concentration (1\% sucrose wt./wt.) as less sweet than did controls. Group effects were only marginal at $0 \mathrm{~g}$ fat, $t(30)=1.75, p<0.05$ (one-tailed) and 3 $\mathrm{g}$ fat, $t(30)=1.67, p<0.10$; but were significant at $7 \mathrm{~g}$ fat, $t(30)=2.25, p<0.025$. No differences between subject groups were observed at $5 \%$ sugar or above. These findings are consistent with the notion that frequent vomiting and repeated exposure of the oral cavity to stomach acids may alter the perception of low-intensity sweet stimuli by bulimic women [14].

All subjects were capable of evaluating increasing fat content in unsweetened stimuli, as summarized in Fig. 2. However, their judgments were progressively less reliable as the sucrose content of the mixture was increased. Analysis of variance showed a main effect of fat, $\mathrm{F}(2,60)=11.93, p<0.01$, and a main effect of sugar, $F(4,120)=7.63, p<0.01$. Again, there was no main effect of subject group, and no grouprelated interactions.

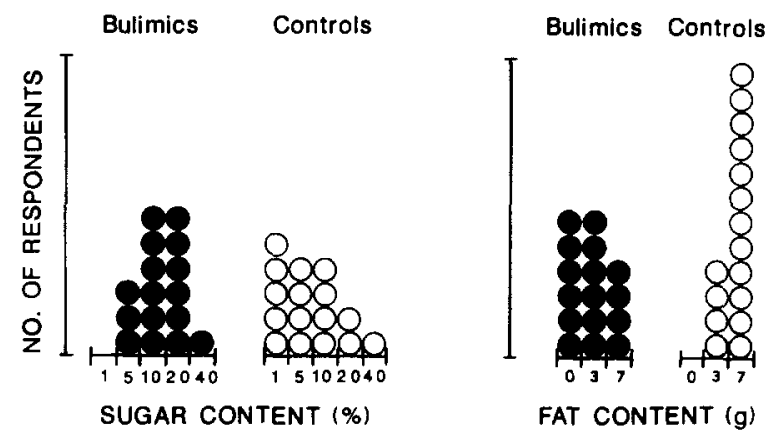

FIG. 4. Frequency distribution of maximum hedonic scores as a function of stimulus sugar and fat content.

TABLE 5

OPTIMALLY PREFERRED SUCROSE AND FAT LEVELS AS PREDICTED BY RSM

\begin{tabular}{lcc}
\hline & $\begin{array}{c}\text { Fat } \\
(\mathrm{g} / 100 \mathrm{~g})\end{array}$ & $\begin{array}{c}\text { Sugar } \\
(\%)\end{array}$ \\
\hline Bulimics & $2.7(0.7)$ & $16.7(3.4)$ \\
Controls & $6.0(0.4)$ & $9.4(3.0)$ \\
\hline
\end{tabular}

The data are means and SEMs (in parentheses).

Pleasantness (hedonic) ratings are summarized in Fig. 3 Hedonic responses were strongly interactive and depended on the relative proportions of the two ingredients-sugar and fat. Analysis of variance showed main effects of both sucrose, $F(4,120)=7.11, p<0.01$, and fat levels, $F(2,60)=4.30$, $p<0.05$. The two groups of subjects differed chiefly in their sensory preferences for fat: bulimic patients preferred stimuli with a lower fat content relative to normal controls. The interaction between diagnostic group and fat content was significant, $\mathrm{F}(2,60)=4.85, p<0.05$

\section{Individual Responses}

Hedonic preference functions that have been averaged across subject groups provide no indication of inter-subject variability. Consequently, further analyses addressed taste response profiles of individual subjects. Frequency distributions of maximum hedonic scores as a function of stimulus sugar and fat content are shown in Fig. 4, separately for bulimic women and normal controls. More bulimic patients preferred intensely sweet stimuli than did controls, Chisquare $(2)=6.66, p<0.05$. In contrast, control subjects overwhelmingly preferred stimuli with a higher fat content: their modal maximum response was for stimuli containing $7 \mathrm{~g}$ fat, Chi-square $(2)=10.4, p<0.01$. It should be noted, however, that there was considerable overlap between the two groups and that it is impossible to assign a subject to a given diagnostic category solely on the basis of her preferences for sugar or fat.

\section{Response Surface Method}

Hedonic responses of individual subjects were also modelled using the Response Surface Method (RSM). This tech- 
BULIMICS

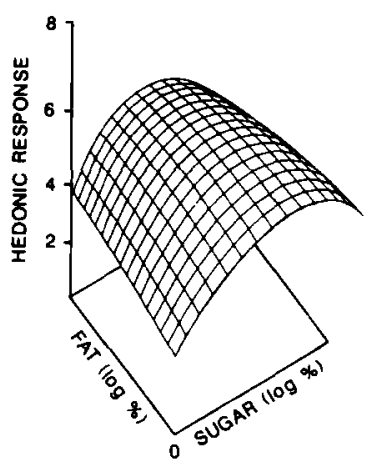

BULIMICS

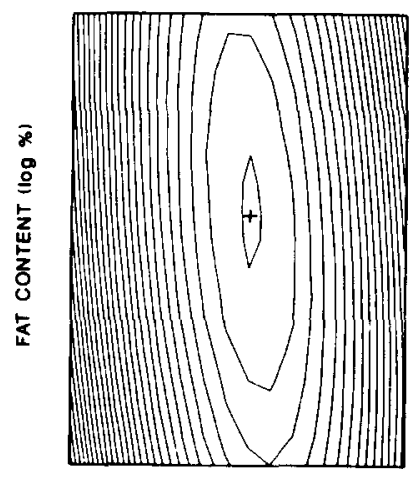

SUGAR CONTENT $(\log \%)$

FIG. 5. Hedonic response surfaces expressed in terms of three dimensional projections (top) and iso-hedonic contours (bottom). The axes represent sucrose ( $x$-axis) and fat content ( $y$-axis) expressed as log percentages wt./wt. Regions of optimum preference as predicted by RSM are denoted by + signs.

nique maps the location of hedonic optima and can be used to predict ingredient composition of stimuli maximally liked by individual subjects.

In the present procedure, RSM was used to derive optimal sucrose and fat levels for individual subjects. These data were then averaged across subject groups, and means and standard errors are summarized in Table 5. Bulimic patients as a group preferred more intensely sweet stimuli $(16.7 \%$ sucrose) than did controls ( $9.4 \%$ sucrose). In contrast, control subjects preferred stimuli with a higher fat content. The best-liked fat level, as predicted by RSM, was $6.0 \mathrm{~g}$ fat per $100 \mathrm{~g}$ for controls, but only $2.7 \mathrm{~g}$ fat per $100 \mathrm{~g}$ for bulimic patients. These levels correspond to $33.3 \%$ and $18.4 \%$ fat as percent solids wt./wt. respectively.

The shapes of hedonic response surfaces for bulimic patients and controls are shown in Fig. 5. The data are presented as three-dimensional projections of the response surface for each subject group (top panel) and as corresponding iso-hedonic contours. The axes represent sucrose ( $x$-axis) and fat content (y-axis) of the stimuli, expressed as log percentages wt./wt. Regions of optimum preference, as determined by the RSM model are denoted by + signs.

\section{DISCUSSION}

Patients with eating disorders show no evidence of deficits in whole mouth sensory perception of stimulus sweetness or fat content, as measured by category scaling of stimuli well above threshold levels. However, in the perception of low-intensity sweet stimuli, bulimic patients sometimes gave slightly lower ratings than did controls. Previous investigators have noted selective taste deficits in bulimia; however, these results were obtained chiefly for threshold sensitivity to sour and bitter tastes [14].

Emaciated anorectic patients have been reported to show elevated preferences for sweet taste $[9,11]$. In previous studies, both anorectic and bulimic patients liked intensely sweet stimuli (approximately 12-15\% sucrose) but showed reduced sensory preferences for fat relative to normalweight controls [11]. In the present study, normal-weight bulimic women liked intensely sweet stimuli (mean optimum: $16.7 \%$ sugar) and were less likely to express preferences for stimuli high in fat content than were normal controls.

The present use of white cheese ("fromage blanc") extends to a new sensory system previous studies that were conducted with mixtures of milk, cream and sugar. It should be noted that the present range of fat levels $(0-7 \mathrm{~g}$ fat per 100 g) was much narrower than the range used in previous studies $(0-52 \mathrm{~g}$ fat per $100 \mathrm{~g})$. However, the new stimuli have the distinct advantage of being comparable in terms of their texture, viscosity and mouthfeel. Interestingly, sensory preferences changed as a function of fat content, even when the fat content itself was only imperfectly monitored by the subjects. These data suggest that fat often provides "hidden calories' in the sense that subjects are often not consciously aware that they are consuming fat-containing foods.

Another issue addressed by the present study concerns individual hedonic response data. Several investigators have pointed out that the "average" hedonic function is likely to be a composite of many, often distinct types of preference response. Booth [5], Pangborn [20] and other investigators $[6,7]$ have argued that sensory studies should focus on individual patterns of taste responsiveness rather than on averaged group data. Such focus on the individual is especially important in clinical studies. Patient populations, whether anorectic or obese, tend to be heterogenous, and are unlikely to present a uniform profile of sensory responsiveness or food choice. The present approach has been to present and analyze hedonic response data for individual subjects in addition to group means. We should also note that our subjects were of a different culture than the typical research sample of Anglo-Saxon university students and were likely to have different eating habits and experiences. In this modest way, our study provides some cross-cultural validation of prior hypotheses about food preferences in bulimia.

What factors account for increased sensory preferences for sweet taste among normal-weight bulimics is still a matter for speculation. One plausible central mechanism involves the endogenous opioid system. Opioids are known to mediate the biological response to stress [17,24] and are thought to modulate the pleasure response to sweetness [4]. Dieting anorectic and bulimic patients have been reported to show both elevated levels of beta-endorphin [17] and elevated preferences for the taste of sweet solutions $[9,11]$. It remains to be seen whether individual taste responsiveness mediates the presumed link between physiological factors, binge-eating behaviors, and the selection of sweet foods during an eating birge. 


\section{ACKNOWLEDGEMENT}

Supported by NIH Grants AM38073, AM37011. Preliminary report based on these data has been presented at FASEB Annual Meeting, Washington, DC, 1987.

\section{REFERENCES}

1. Abraham, S. F. and P. J. V. Beaumont. How patients describe bulimia or binge eating. Psychol Med 12: 625-635, 1982.

2. American Psychiatric Association Committee on Nomenclature and Statistics. Diagnostic and Statistical Manual of Mental Disorders, third edition (revised). Washington. DC: American Psychiatric Association, 1987.

3. Beaumont, P. J. V. and T. L. Chambers. The diet composition and nutritional knowledge of patients with anorexia nervosa. $J$ Hum Nutr 35: 265-273, 1981.

4. Blass, E. M. Opioids, sugar and the inherent taste of sweet: Broad motivational implications. In: Sweetness, edited by J. Dobbing. Berlin: Springer-Verlag, 1987.

5. Booth, D. A., M. T. Conner and S. Marie. Sweetness and food selection: Measurement of sweeteners' effect on acceptance. In: Sweetness, edited by J. Dobbing. Berlin: Springer-Verlag, 1987.

6. Drewnowski, A. New techniques: Multidimensional analysis of taste responsiveness. Int J Obes 8: 599-607, 1984.

7. Drewnowski, A. Sweetness and obesity. In: Swcetness, edited by J. Dobbing. Berlin: Springer-Verlag, 1987.

8. Drewnowski, A., J. B. Brunzell, K. Sande, P. H. Iverius and M. R. C. Greenwood. Sweet tooth reconsidered: Taste responsiveness in human obesity. Physiol Behav 35: 617-622, 1985.

9. Drewnowski, A. M. R. C. Greenwood and K. A. Halmi. Carbohydrate or fat phobia: Taste responsiveness in anorexia nervosa. Fed Proc 43: 475, 1984.

10. Drewnowski, A. and M. R. C. Greenwood. Cream and sugar: Human preferences for high-fat foods. Physiol Behav 30: 629 633.1983.

11. Drewnowski, A., K. A. Halmi, B. Pierce, J. Gibbs and G. P. Smith. Taste and eating disorders. Am J Clin Nutr 46: 442 $450,1987$.

12. Drewnowski, A., D. Yee and D. D. Krahn. Bulimia on campus: Incidence and recovery rates. American Psychiatric Association Annual Meeting, Chicago, IL, 1987.
13. Hurst, P. S., J. H. Lacey and A. H. Crisp. Teeth, vomiting and diet: a study of the dental characteristics of seventeen anorexia nervosa patients. Postgrad Med J 53: 298-305, 1977.

14. Jirik-Babb, P. and J. L. Katz. Taste perception in anorexia nervosa and bulimia. Presented at 2nd International Conference on Eating Disorders, New York, 1986.

15. Johnson, C., C. Lewis and J. Hagmann. The syndrome of bulimia: Review and synthesis. Psychiatr Clin North Am 7: 247-272, 1984.

16. Johnson, C. L., M. K. Stuckey, L. D. Lewis and D. M. Schwartz. Bulimia: a descriptive survey of 316 cases. Int J Eating Disorders 2: 3-16, 1982.

17. Kaye, W. H., D. Pickar, D. Naber and M. H. Ebert. Cerebrospinal fluid opioid activity in anorexia nervosa. Am $J$ Psychiatry 139: 643-645, 1982

18. Mawson. A. R. Anorexia nervosa and the regulation of intake: A review. Psuchol Med 4: 289-308, 1974.

19. Mitchell, J, E. and D. C. Laine. Monitored binge-eating behavior in patients with bulimia. Int J Eating Disorders 4: 177-183, 1985.

20. Pangborn, R. M. and S. D. Pecore. Taste perception of sodium chloride in relation to dietary intake of salt. Am J Clin Nutr 35: $510-520,1982$.

21. Polivy, J. and C. P. Herman. Dieting and binging: a causal analysis. Am Psychol 40: 193-201, 1985.

22. Rosen, J. C., H. Leitenberg, C. Fisher and C. Khazam. Bingeeating episodes in bulimia nervosa: The amount and type of food consumed. Itt J Eating Disorders 5: 255-267. 1986.

23. Russell, G. F. M. The nutritional disorder in anorexia nervosa. $J$ Psychosom Res 11: 141-149, 1967.

24. Weiner, H. The physiology of eating disorders. Int $J$ Eating Disorders 4: $347-388,1985$ 